\title{
Multiple system atrophy: a disorder targeting the brainstem control of survival
}

\author{
Eduardo E. Benarroch ${ }^{1}$
}

Received: 7 October 2019 / Accepted: 8 October 2019 / Published online: 24 October 2019

(c) Springer-Verlag GmbH Germany, part of Springer Nature 2019

Keywords MSA · C1 area - Orthostatic hypotension · Pontine micturition center · Medullar raphe $\cdot$ Parabrachial nucleus · Synuclein

\section{Introduction}

Multiple system atrophy (MSA) is a neurodegenerative synucleinopathy characterized by severe autonomic failure, manifested primarily by orthostatic hypotension $(\mathrm{OH})$, neurogenic bladder and sexual dysfunction associated with parkinsonism, ataxia, or both. One salient feature of MSA is the coexistence of respiratory dysfunction (including sleep apnea and laryngeal stridor) and rapid eye movement (REM) sleep behavior disorder. This indicates that MSA neuropathology selectively targets brainstem areas participating in the integrative control of autonomic, respiratory, and arousal/sleep functions. Recently, rodent studies using selective activation or inactivation of specific brainstem neurons groups have provided further insight into the mechanisms controlling these vital functions and support the neuropathological evidence of extensive brainstem involvement in MSA.

\section{Baroreflex dysfunction}

One characteristic manifestation of MSA is neurogenic $\mathrm{OH}$, which reflects impaired baroreflex efferent control of blood pressure via sympatho-excitatory input to splanchnic and skeletal muscle blood vessels. This output is activated by premotor glutamatergic sympatho-excitatory neurons in the rostral ventrolateral medulla (RVLM), including the C1 epinephrine-synthesizing group. In MSA, there is consistent loss of $\mathrm{C} 1$ neurons, which is typically more severe than

Eduardo E. Benarroch

Benarroch.eduardo@mayo.edu

1 Department of Neurology, Mayo Clinic Rochester, Rochester, MN, USA the one observed in other synucleinopathies such as Parkinson disease [9]. Although there is loss of preganglionic sympathetic neurons in MSA, there is relative preservation of peripheral sympathetic innervation, at least in the initial stages of the disease. Loss of $\mathrm{C} 1$ neurons is associated with abundant accumulation of $\alpha$-synuclein-containing glial cytoplasmic inclusions (GCI) although there appears to be no correlation between neuronal loss and GCI accumulation in the $\mathrm{C} 1$ area [10]. Optogenetic studies in mice have shown that, $\mathrm{C} 1$ neurons are not critical for tonic maintenance of arterial pressure during wakefulness, but they mediate arterial blood pressure increase following baroreceptor denervation, and have a major role in sympatho-excitatory responses to hypoxia or other internal stressors $[1,11]$. $\mathrm{C} 1$ neurons also promote respiration via their projections to the retrotrapezoid nucleus and lateral parabrachial nucleus and behavioral arousal via their projections to the locus coeruleus. Another important feature of MSA is impaired arginine vasopressin (AVP) release in response to orthostatic stress or hypovolemia. In normal conditions, inputs from baroreceptors and volume receptors acting on the NTS tonically inhibit AVP release from magnocellular neurons in the supraoptic (SON) and paraventricular nuclei (PVN). The AVP increase during baroreflex withdrawal, as occurs during orthostatic stress is, in part, mediated by a group of noradrenergic neurons in the A1 area of the caudal ventrolateral medulla. Neuropathological evidence showed that there is preservation of magnocellular neurons in the SON. However, there is loss of A1 neurons and noradrenergic fibers innervating these AVP-secreting cells. This provides a putative substrate for the impaired reflex AVP release in MSA [7]. 


\section{Neurogenic bladder}

Neurogenic bladder is a major and oftentimes presenting feature of MSA. Common to many other neurodegenerative disorders, detrusor overactivity producing urinary urgency can be an initial feature. However, unlike other disorders, urinary retention and overflow incontinence (i.e., underactive bladder) are salient features of MSA. The normal micturition reflex involves several areas of the CNS, including the ventrolateral periaqueductal gray (vlPAG) that receives bladder afferents, and the pelvic organ control center (also called pontine micturition center, PMC), which send descending projections to the sacral spinal cord to elicit coordinated activation of sacral parasympathetic neurons triggering the contraction of the detrusor muscle, while inhibiting somatomotor neurons of the Onuf nucleus, which maintain contraction of the external urethral sphincter. The output neurons of this reflex, including the sacral preganglionic and Onuf motoneurons, have long been recognized to be affected in MSA. In addition, there is evidence of loss of corticotrophin releasing factor (CRF) neurons in the PMC, which may additionally explain the impairment of this reflex [2]. Accumulation of GCI in the vlPAG may also contribute to impaired control of the micturition reflex [5].

\section{Impaired vagal control of visceral organs}

In MSA, like in Parkinson disease, there is loss of cholinergic neurons in the dorsal motor nucleus of the vagus, which primarily control upper gastrointestinal motility [8]. There is also loss of cholinergic neurons in the caudal ventrolateral portion of the nucleus ambiguus, which provides the major vagal influence to the sinus node. This can explain the impaired respiratory modulation of heart rate observed in MSA patients. This portion of the nucleus ambiguus also contains neurons that innervate dilator muscles of the upper airway, and their involvement may contribute to laryngeal stridor, at least in some cases.

\section{Respiratory manifestations of MSA and their putative substrates}

Respiratory manifestations, including sleep apnea, respiratory dysrhythmia, and laryngeal stridor are prominent manifestation of MSA. One potential substrate for central sleep apnea and respiratory dysrhythmia in MSA is loss of neurons in the pre-Bötzinger complex, which is the inspiratory pattern generator. Loss of these neurons was first suggested by using neurokinin-1 immunoreactivity as a marker [3], and later confirmed with studies using the more selective marker somatostatin [13]. There is also profound loss of serotonergic neurons in the medullary raphe, including the nucleus raphe obscurus and nucleus raphe magnus in MSA $[4,10]$. These nuclei have an important role in respiratory chemosensitivity and facilitation of respiratory motoneuron activity. These findings thus provide a potential mechanism for some of the respiratory manifestations of MSA. Consistent with these findings, loss of medullary serotonergic neurons is more marked in those MSA patients that suffered sudden death during sleep [14]. The pathophysiology and anatomic substrates of laryngeal stridor in MSA are incompletely understood. In some cases, stridor may reflect weakness of laryngeal abductor muscles. However, in other cases, there is documented paradoxical activation of laryngeal adductor muscles in inspiration (i.e., dystonia). Neurons of the parabrachial nuclear complex participate in coordinating respiratory pump activity with that of muscles controlling the upper airway. There is loss of neurons in both the lateral and medial parabrachial nuclei in MSA [6], which can contribute to laryngeal stridor and respiratory dysrhythmia. Both cholinergic and monoaminergic medullary groups affected in MSA also control upper airway resistance as described above. The lateral parabrachial nucleus is also critically involved in arousal from sleep in the setting of hypercapnia [12]. To what extent involvement of this nucleus predisposes to sudden death during sleep, a common cause of death in MSA, is an intriguing possibility that need to be further explored.

In summary, MSA is a prototypical disorder affecting interconnected brainstem areas involved in vital functions such as cardiovascular and respiratory control, micturition, and behavioral arousal. Recent experimental studies using a variety of genetic, physiological, and neuroanatomic tools have provided important new insight in how these areas participate in these functions, and validate the putative clinic-pathological correlations described above. Furthermore, the anatomical connectivity between the brainstem areas involved in cardiovascular, respiratory, and arousal function, as exemplified by the $\mathrm{C} 1$ area, support the concept of "prion-like" propagation of disease neuropathology. Thus, clinico-pathological studies in MSA have provided valuable information on the central organization of control of survival in humans.

Acknowledgements This short article is a summary of the Streeten Plenary Lecture that the author gave at the 30th International Symposium of the Autonomic Nervous System in November 2019.

Funding None. 


\section{Compliance with ethical standards}

Conflict of interest Eduardo E. Benarroch declares that there is no conflict of interest.

\section{References}

1. Abbott SB, Coates MB, Stornetta RL, Guyenet PG (2013) Optogenetic stimulation of $\mathrm{c} 1$ and retrotrapezoid nucleus neurons causes sleep state-dependent cardiorespiratory stimulation and arousal in rats. Hypertension 61:835-841

2. Benarroch EE, Schmeichel AM (2001) Depletion of corticotrophin-releasing factor neurons in the pontine micturition area in multiple system atrophy. Ann Neurol 50:640-645

3. Benarroch EE, Schmeichel AM, Low PA, Parisi JE (2003) Depletion of ventromedullary NK-1 receptor-immunoreactive neurons in multiple system atrophy. Brain 126:2183-2190

4. Benarroch EE, Schmeichel AM, Low PA, Parisi JE (2004) Involvement of medullary serotonergic groups in multiple system atrophy. Ann Neurol 55:418-422

5. Benarroch EE, Schmeichel AM, Low PA, Parisi JE (2010) Differential involvement of the periaqueductal gray in multiple system atrophy. Auton Neurosci Basic Clin 158:111-117

6. Benarroch EE, Schmeichel AM, Low PA, Parisi JE (2013) Parabrachial nucleus involvement in multiple system atrophy. Auton Neurosci Basic Clin 177:170-174
7. Benarroch EE, Schmeichel AM, Sandroni P, Low PA, Parisi JE (2006) Differential involvement of hypothalamic vasopressin neurons in multiple system atrophy. Brain 129:2688-2696

8. Benarroch EE, Schmeichel AM, Sandroni P, Low PA, Parisi JE (2006) Involvement of vagal autonomic nuclei in multiple system atrophy and Lewy body disease. Neurology 66:378-383

9. Benarroch EE, Smithson IL, Low PA, Parisi JE (1998) Depletion of catecholaminergic neurons of the rostral ventrolateral medulla in multiple systems atrophy with autonomic failure. Ann Neurol 43:156-163

10. Coon EA, Schmeichel AM, Parisi JE, Cykowski MD, Low PA, Benarroch EE (2016) Medullary neuronal loss is not associated with alpha-synuclein burden in multiple system atrophy. Mov Disord 31:1802-1809

11. Guyenet PG, Stornetta RL, Bochorishvili G, Depuy SD, Burke PG, Abbott SB (2013) C1 neurons: the body's EMTs. Am J Physiol Regul Integr Comp Physiol 305:R187-R204

12. Kaur S, Saper CB (2019) Neural circuitry underlying waking up to hypercapnia. Front Neurosci 13:401

13. Schwarzacher SW, Rub U, Deller T (2011) Neuroanatomical characteristics of the human pre-Botzinger complex and its involvement in neurodegenerative brainstem diseases. Brain 134:24-35

14. Tada M, Kakita A, Toyoshima Y, Onodera O, Ozawa T, Morita T, Nishizawa M, Takahashi H (2009) Depletion of medullary serotonergic neurons in patients with multiple system atrophy who succumbed to sudden death. Brain 132:1810-1819 